IN3 Working Paper Series INTERNET INTERDISCIPLINARY INSTITUTE

\title{
Procesos psicoeducativos en el aprendizaje cooperativo. Dimensiones para el análisis en un escenario educativo presencial con tecnología.
}

Lorena Becerril Balín (Ibecerril@uoc.edu)

IN3-UOC (PhD Fellow)

Working Paper Series DWP10-001

Grupo de investigación: Sinte (Seminari Interuniversitari d'Estratègies d'Aprenentatge)

Coordinador del grupo de investigación: Carles Monereo Font

Fecha de recepción: 2010

Fecha de aceptación: 2010

Fecha de publicación: 2011

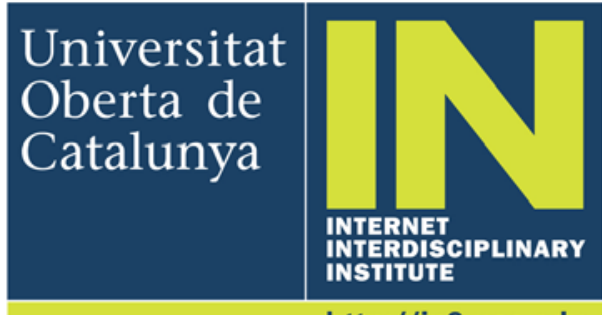




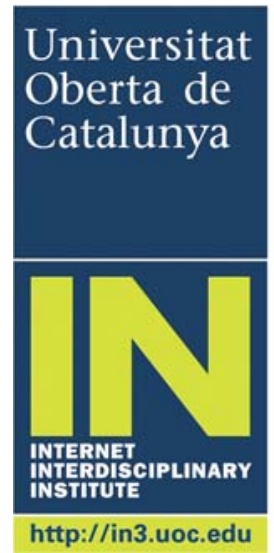

Internet Interdisciplinary Institute (IN3)

http://www.in3.uoc.edu

Edifici MediaTIC

c/ Roc Boronat, 117

08018 Barcelona

Espanya

Tel. 934505200

Universitat Oberta de Catalunya (UOC)

http://www.uoc.edu/

Av. Tibidabo, 39-43

08035 Barcelona

Espanya

Tel. 932532300

The texts published in this publication are - unless indicated otherwise - covered by the Creative Commons Spain Attribution-Non commercial-No derivative works 3.0 licence. You may copy, distribute, transmit and broadcast provided that you attribute it (authorship, publication name, publisher) in the manner specified by the author(s) or licensor(s).

The full text of the licence can be consulted here:

http://creativecommons.org/licenses/by-nc-nd/3.0/es/deed.en. 


\section{Sumario}

Resumen 5

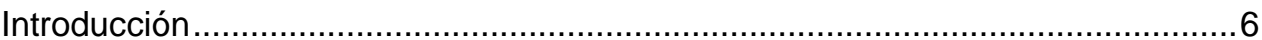

1. Procesos psicoeducativos en el aprendizaje cooperativo ............................... 7

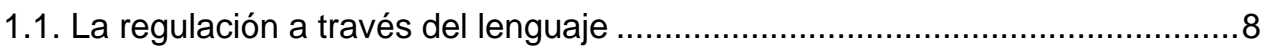

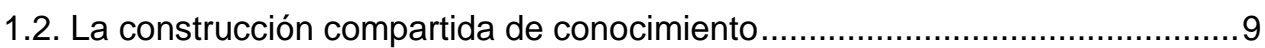

1.3. Controversias o conflictos entre puntos de vista moderadamente divergentes 10

1.4. La construcción de representaciones compartidas....................................11

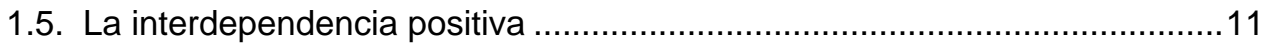

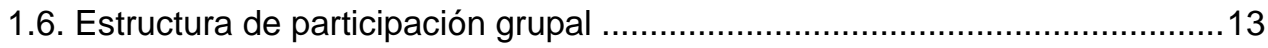

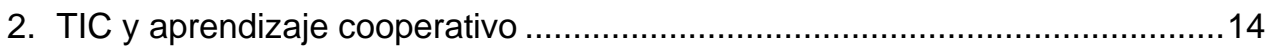

3. Situaciones educativas de aprendizaje cooperativo presencial con TIC.........16

4. Una propuesta de dimensiones de análisis .................................................18

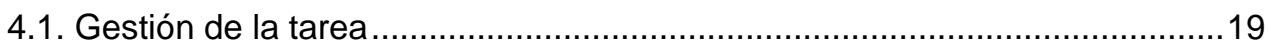

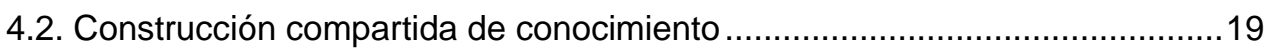

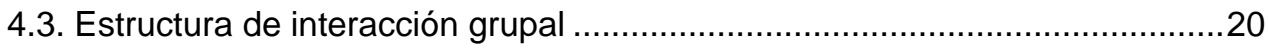

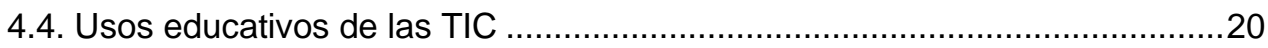

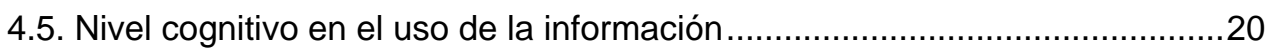

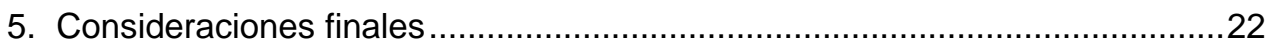

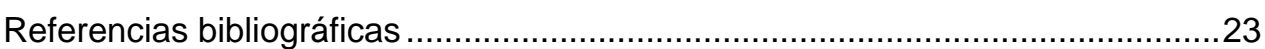




\section{Procesos psicoeducativos del aprendizaje cooperativo. Dimensiones para el análisis en un escenario educativo presencial con tecnología}

Lorena Becerril Balín (Ibecerril@uoc.edu)

IN3-UOC (PhD Fellow)

\section{Recommended citation:}

BECERRIL, Lorena (2011). "Procesos psicoeducativos del aprendizaje cooperativo. Dimensiones para el análisis en un escenario presencial con tecnología" [online doctoral working paper]. (Working Paper Series; DWP10-001). IN3 Working Paper Series. IN3 (UOC). [Accessed: dd/mm/yy].

$<$ http://in3wps.uoc.edu/ojs/index.php/in3-working-paper-series/article/view/n10becerril/n10-becerril> 


\section{Resumen}

La finalidad de este artículo es presentar una propuesta de dimensiones de análisis de los procesos psicoeducativos implicados en el aprendizaje cooperativo, en un escenario educativo donde se hace un uso educativo intensivo de las TIC. La elaboración de esta propuesta implica, en primer lugar, la revisión teórica previa de los procesos psicoeducativos que explican la eficacia del proceso del aprendizaje cooperativo y, en segundo lugar, la definición de un posible escenario educativo dónde aplicar esta propuesta de análisis. Una vez identificados estos procesos y descrito dicho escenario dispondremos de un marco teórico y práctico del cual partir para tomar decisiones en relación a las dimensiones de análisis que incluimos en nuestra propuesta.

Palabras clave:

Aprendizaje cooperativo, competencias básicas, usos educativos de las TIC, procesos psicoeducativos 


\section{Introducción}

El nuevo escenario de la educación obligatoria resultado de la propuesta curricular competencial que propone la Ley Orgánica de Educación 2/2006, de 2 de mayo de 2006, concretado en Cataluña en el Decreto 143/2007 de 26 de junio y cuya aplicación finalizó el curso 2009-2010, conlleva varios retos educativos que se derivan de la nueva definición de currículum como "el conjunto de objetivos, competencias básicas, contenidos, métodos y criterios de evaluación de cada uno de las enseñanzas reguladas por la presente ley" (LOE).

La necesidad de desarrollar dicho currículum competencial refuerza la importancia del aprendizaje cooperativo y del uso educativo de las Tecnologías de la Información y la Comunicación en tanto que competencias metodológicas que deben integrarse en el currículum. En este sentido, el aprendizaje cooperativo nos remite a la llamada competencia de aprender a aprender, que implica disponer de habilidades para guiar el propio aprendizaje, y el uso de las TIC a la competencia del tratamiento de la información y la competencia digital. Dicha competencia incorpora diferentes habilidades que van desde el acceso a la información hasta su transmisión utilizando diversos soportes, incluyendo la utilización de las TIC como elemento esencial para informarse, aprender y comunicarse.

Aunque la implantación de dicha ley no supone una novedad en cuanto a las ventajas del aprendizaje cooperativo en la enseñanza presencial, sí que promueve explícitamente su utilización. La descripción de las bondades educativas del aprendizaje cooperativo es una tarea ya llevada a cabo por muchos autores a partir de la creencia de que el aprendizaje se incrementa cuando los estudiantes desarrollan destrezas cooperativas para aprender y solucionar problemas en un contexto de interacción entre iguales (Ellis, Klahr y Siegler, 1993; Azmitia, 1988).

En este sentido, después de revisar las investigaciones llevadas a cabo hasta el momento y que se corresponden, según Melero y Fernández (1995), con la primera generación de estudios sobre aprendizaje cooperativo, Slavin (1980) concluyó, que si bien el aprendizaje cooperativo no es peor para el rendimiento académico que el tradicional, lo supera en cuanto a la efectividad para alumnos con bajo rendimiento. Además también se aprecian ventajas en relación a la adquisición de capacidades cognitivas complejas, así como beneficios en las relaciones interraciales y en el refuerzo del sentimiento de pertenencia del alumnado a la escuela. Igualmente, otros autores (Gabbert, Johnson y Johnson, 1986) concluían de sus investigaciones que los estudiantes solucionan los problemas más correctamente cuando colaboran con otros, especialmente cuando se trata de una tarea conceptual y compleja.

Si nos remitimos a la denominada segunda generación de investigaciones sobre aprendizaje cooperativo, dónde situamos nuestra propuesta, el objetivo no es valorar qué método es el mejor para aumentar el rendimiento académico sino identificar y explicar cuáles son las causas y los mecanismos que explican los resultados 
obtenidos del aprendizaje cooperativo. Nuestra atención se centra específicamente en los procesos psicoeducativos que pueden explicar la eficacia del proceso educativo que implica el aprendizaje cooperativo.

Además, de acuerdo con otros autores que han considerado las nuevas tecnologías en relación al aprendizaje cooperativo (véase Koschmann (1996) con su propuesta de CSCL-Computer Supported Collaborative Learning, marco que recoge en nuestro país Gros (2001). Igualmente y en escenarios educativos presenciales, Wegerif (1996) y Thompson (2006), centrados en la influencia del ordenador en el habla a partir de la utilización de diferentes programas educativos), nos interesa saber qué ocurre cuando las TIC están presentes en escenarios de aprendizaje cooperativo y qué papel juegan en dichos procesos.

Para lograr este propósito, dedicaremos un primer apartado de este documento a describir los procesos psicoeducativos que subyacen en el aprendizaje cooperativo, un segundo apartado la contribución de la tecnología al aprendizaje cooperativo, un tercer apartado a caracterizar el escenario educativo susceptible de ser objeto de investigación, y finalmente, un cuarto apartado donde concretaremos las dimensiones y categorías relevantes para el análisis de acuerdo con las características educativas del escenario educativo descrito.

\section{Procesos psicoeducativos en el aprendizaje cooperativo}

Tomar en consideración los procesos psicoeducativos que subyacen en el aprendizaje cooperativo supone tomar partido ante la dualidad tantas veces establecida por la Psicología de la Educación entre la dimensión cognitiva y la dimensión social del ser humano. De acuerdo con algunos autores (Coll y Miras, 1990; Duran, 2002), no sólo hay que superar esta dualidad entre lo que es cognitivo y lo que es social sino que hay que interrelacionar ambas dimensiones de manera que la cognición y la interacción social sean vistas como elementos dinámicos e interactivos que se construyen y reconstruyen durante el proceso educativo.

Por otro lado hay que tener en cuenta que todavía hoy, el proceso concreto que desencadena el aprendizaje en situaciones colaborativas no ha recibido una única y reconocida explicación de cómo se produce. Mientras unos investigadores han propuesto algunos factores responsables de la mejora del aprendizaje como el conflicto sociocognitivo (Doise et al., 1975) o el aumento de la verbalización (Teasley, 1992), otros se han encargado de contradecir dichos factores mostrando que el efecto de la colaboración no puede atribuirse a un sólo factor. Puesto que desde nuestro punto de vista los procesos psicoeducativos que tienen lugar en el aprendizaje cooperativo son variados y por sí solos no ofrecen una explicación válida, consideramos necesario tratarlos de manera integrada, esto es, teniendo en cuenta tanto la dimensión individual como la dimensión social de cada proceso, a fin de 
entender cómo se produce el aprendizaje del estudiante tanto en el plano intrapsicológico como interpsicológico.

Dicho esto, nos centramos en seis procesos psicoeducativos que a nuestro modo de ver son los más relevantes en el aprendizaje cooperativo:

- la regulación a través del lenguaje

- la construcción compartida del conocimiento

- el conflicto entre puntos de vista moderadamente divergentes

- la construcción de representaciones compartidas

- la interdependencia positiva

- la estructura de participación que se establece entre los participantes.

A continuación se presentan las bases teóricas de dichos procesos sociocognitivos desde los diferentes enfoques de la enseñanza y el aprendizaje, así como los elementos a tener en cuenta en nuestra propuesta de dimensiones de análisis.

\subsection{La regulación a través del lenguaje}

Uno de los elementos fundamentales de la interacción entre alumnos es el estudio del lenguaje como instrumento de co-regulación. Desde la perspectiva sociocultural el interés recae sobre la co-regulación definida por Coll (1984) como la utilización del lenguaje para regular las propias acciones y las de los compañeros a la vez que se es regulado por el lenguaje de los otros, produciéndose de este modo una regulación mutua entre los miembros que participan en la interacción social.

Asimismo, otro aspecto a destacar es la importancia de la toma de conciencia del propio conocimiento que las situaciones cooperativas promueven. En este sentido, tal como apunta Crook (1998), cuando el alumno verbaliza y hace público y explícito su pensamiento debe organizar su conocimiento en beneficio de la actividad conjunta. Igualmente, Dillenbourg (1999) hace referencia a la explicación a los otros como un proceso psicoeducativo que emerge en situaciones colaborativas y que promueve el aprendizaje en tanto que implica la identificación del conocimiento que el individuo requiere para completar sus propias explicaciones.

Si bien desde la perspectiva sociocultural el estudio del lenguaje como instrumento de mediación ha tomado un papel preeminente, otras perspectivas también han dado cabida al estudio de los procesos regulativos. En efecto, mientras que desde una perspectiva más sociocognitiva el proceso de regulación se define en términos de autorregulación, es decir, como un proceso individual que tiene lugar en cada individuo a fin de favorecer el aprendizaje estratégico (Schunk y Zimmerman, 1994), desde posicionamientos próximos a la cognición distribuida la regulación se considera colectiva, a saber, los miembros del grupo desarrollan una conciencia compartida de los objetivos y de la tarea que les permite construir conjuntamente el proceso regulador. 


\subsection{La construcción compartida de conocimiento}

Desde las perspectivas sociocognitiva y sociocultural del aprendizaje se considera un aspecto clave el análisis del proceso de construcción colaborativa de conocimiento. Para describir dicho proceso se utilizan diferentes términos como co-construcción de conocimiento, construcción compartida de conocimiento, construcción colaborativa de conocimiento o construcción conjunta de conocimiento. Como advierte Coll (1996), a menudo estas denominaciones van más allá de aspectos que hacen referencia únicamente al contenido como las metas o los roles en la interacción.

Entender cómo se construye el conocimiento en las interacciones de los grupos cooperativos y cómo evoluciona durante el proceso de enseñanza y aprendizaje requiere dar sentido a las conversaciones que tienen los alumnos y a las acciones que llevan a cabo. De acuerdo con Coll y Onrubia (1996), vincular el discurso y la construcción de significados compartidos deviene fundamental para entender lo que pasa tanto interpsicológicamente en la interacción social como intrapsicológicamente en los individuos.

Otro proceso psicoeducativo estrechamente ligado a la construcción compartida de conocimiento es la intersubjetividad, que supone comprender y adoptar el marco de referencia del otro. Un requisito fundamental para que sea posible la actividad conjunta es la existencia de una comprensión mutua entre los participantes. Dicha comprensión mutua implica tener unas referencias comunes a partir de las cuales definir la situación. Dillenbourg (1999) la define como la construcción conjunta de la comprensión mutua, Crook (1998) como un objeto cognitivo construido por los participantes y Wertsch (1988) habla de la existencia de intersubjetividad a diferentes niveles o grados.

El esfuerzo que requiere lograr ciertos niveles de intersubjetividad compartiendo la definición de la situación se manifiesta en la utilización de formas específicas de habla (Mercer, 1997) y en las estrategias discursivas. En relación a los tipos de habla Mercer distingue entre conversación exploratoria, de discusión y acumulativa. En la primera, los estudiantes tratan de forma crítica pero constructiva las ideas de los demás, ofrecen afirmaciones o sugerencias para considerarlas conjuntamente y las justifican explorando hipótesis alternativas. En cambio, en la conversación de discusión los hablantes están en desacuerdo y toman decisiones individualmente. Mientras que en la conversación acumulativa los hablantes construyen positivamente conocimiento sobre las ideas del otro dominando el acuerdo. Según Mercer la conversación exploratoria es la adecuada para resolver problemas conjuntamente y avanzar en la comprensión.

En relación a las estrategias discursivas, cabe mencionar la propuesta de Hogan, Nastasi y Pressley (2000) que clasifican las estrategias según sean conceptuales, metacognitivas o de planteamiento de preguntas. Mientras que las estrategias conceptuales hacen referencia a les presentaciones o repeticiones de ideas, las metacognitivas se refieren a la evaluación de las ideas propias o de los otros y las estrategias de planteamiento de preguntas se relacionan con plantear preguntas 0 solicitar información. 
Otras estrategias discursivas que deberían poner en juego los estudiantes en situaciones colaborativas y que se consideran motor de aprendizaje (Dillenbourg, 1999) son la argumentación y la negociación. La primera de ellas implica la construcción de argumentos y supone una forma específica de comunicación ante la presencia de un conflicto o discrepancia. Argumentar es también evaluar, comparar y contrastar la fuerza o debilidad de diferentes puntos de vista. Además, conlleva una serie de habilidades lingüísticas, especialmente las que se refieren a la elaboración de frases complejas de carácter causal y consecutivo que favorecen el aprendizaje.

En relación a la negociación, considerada por el constructivismo social como un mecanismo de interiorización del conocimiento y por la perspectiva cognitiva como un proceso a través del cual emerge la comprensión mutua, hay que tener en cuenta que aunque ésta se considera estrechamente ligada a la argumentación, no siempre la acompaña. De hecho, tal como apuntan Dillenbourg y Baker (1995), la negociación sólo aparece cuando en la tarea se da un espacio para ella de manera que puede ser inhibida si la tarea propuesta no implica el hecho de ponerse de acuerdo.

\subsection{Controversias o conflictos entre puntos de vista moderadamente divergentes}

La potencialidad constructiva de los conflictos que aparecen entre los alumnos o en ellos mismos, como resultado de la confrontación de diferentes puntos de vista o de niveles diferentes de aprendizaje es un otro proceso psicoeducativo a tomar en consideración. La adopción de diferentes estrategias para resolver las diferencias y superar el desequilibrio que provocan las controversias conlleva un proceso social y cognitivo que puede derivar en aprendizaje. Puntambekar (2006) se centra en dicho proceso para estudiar cómo las perspectivas divergentes en relación con el contenido cambian a través del proceso de colaboración mediante estrategias para resolver las divergencias como la imposición asertiva, el consentimiento o la atribución recíproca de sentido produciéndose la construcción colaborativa de conocimiento.

Aún así, tal como han demostrado diversos estudios, la existencia de la controversia por si sola no garantiza el aprendizaje puesto que se pueden identificar otros factores y variables que pueden influir, como por ejemplo la frecuencia con la que se producen los conflictos y el grado de dificultad de resolución de la controversia (Johnson y Johnson, 1990; Bearison, Magzamen y Filardo, 1986). En cambio, en nuestro país Fernández y Melero (1995) consideran que el tipo de regulación del conflicto, dependiente tanto de aspectos cognitivos como relacionales, como un factor decisivo en la efectividad de los conflictos en el aprendizaje. 


\subsection{La construcción de representaciones compartidas}

La consideración del aprendizaje colaborativo como un proceso de convergencia en el cual los alumnos coinciden gradualmente en significados y logran una representación compartida del conocimiento (Roschelle, 1992) es otro mecanismo que puede explicar cómo se aprende durante el aprendizaje colaborativo. La convergencia tiene lugar porque la naturaleza recíproca de la colaboración permite un aumento en la similitud de las representaciones cognitivas de los participantes.

Sin embargo, tal como apuntan Jeong y Chi (2007) el hecho de estar sometidos a una situación de interacción colaborativa, en la cual los alumnos se ven obligados a compartir los objetivos de la tarea, interpretar conjuntamente la situación y coordinar sus acciones, no es la única fuente de convergencia. En efecto, el hecho de pertenecer a una misma cultura y estar expuestos a la misma experiencia de aprendizaje y a los mismos materiales didácticos también puede contribuir a que se produzca dicha convergencia, de manera que los investigadores deben controlar estas dos formas de convergencia y atribuir a cada una las aportaciones que le correspondan.

Entender la convergencia de conocimiento no sólo como un proceso sino también como un resultado del aprendizaje colaborativo nos remite, al igual que ocurre con el proceso de construcción colaborativa de conocimiento, a diferentes denominaciones que se utilizan para describir los resultados del aprendizaje cooperativo: significados compartidos (shared meanings), conocimiento común (commom knowledge), conocimiento compartido (shared knowledge) o la mencionada convergencia de conocimiento (knowledge convergence). Además, podemos encontrar divergencias en los significados que se les atribuyen. En este sentido y seguiendo a Thompson y Fine (1999), por lo menos existen tres significados diferentes: a) dividido en porciones en el sentido de la teoría de la cognición distribuida (Salomon, Perkins y Globerson, 1991), este significado hace referencia a lo que diferencia o se divide entre los miembros del grupo a menudo como resultado de la adopción de diferentes roles, b) tener en común modelos mentales (Cannon-Bowers, Salas y Converse, 1993), es decir, centrarse en las representaciones comunes de los participantes y c) participar en el acuerdo, relacionado con la noción de consenso y acuerdo y la creación del "commom ground" (Clark y Brennan, 1991).

\subsection{La interdependencia positiva}

Otro proceso fundamental de la cooperación entre iguales ligado a factores motivacionales y relacionales que contribuye a explicar la efectividad del aprendizaje cooperativo es la interdependencia positiva. La interdependencia positiva supone que el éxito de cada miembro está unido al resto del grupo y viceversa, y es precisamente 
la percepción de los estudiantes de esta vinculación uno de los factores que promueve la interacción colaborativa.

A pesar de que hay un acuerdo general en considerar la interdependencia como un elemento clave de la cooperación efectiva, algunos autores difieren en la manera de lograrla, es decir, en las técnicas para promoverla. Mientras Johnson y Johnson (1999), consideran que la clave es establecer objetivos grupales y la división de recursos, Slavin (1990) defiende la recompensa individual de los estudiantes por el trabajo realizado en el grupo como elemento esencial para alcanzarla.

Al margen de dichas diferencias, la interdependencia positiva implica que los alumnos se hacen responsables de completar su parte del trabajo y al mismo tiempo de que los otros miembros logren los objetivos grupales (Johnson y Johnson, 1990), de ahí que cuando se produce la ausencia de interdependencia se produzcan los esfuerzos individuales. Johnson y Johnson (1999) reúnen en dos grupos los diferentes tipos de interdependencia: resultados de interdependencia y medios de interdependencia. Los resultados de interdependencia se producen cuando los alumnos se orientan hacia el resultado deseado, mientras que los medios de interdependencia se refieren a los procedimientos a través de los cuales se logran las metas y las recompensas.

Los resultados de interdependencia incluyen la interdependencia de objetivos y la interdependencia de recompensas. La primera consiste en que los estudiantes sienten que sólo pueden alcanzar sus objetivos si todos los integrantes de su grupo también alcanzan los suyos y la segunda hace referencia a la misma recompensa que reciben los estudiantes por el trabajo grupal realizado.

Los medios de interdependencia que identifican los recursos a través de los cuales los objetivos y las recompensas se logran, comprenden la interdependencia de recursos, la interdependencia de roles y la interdependencia de la tarea. En la interdependencia de recursos cada miembro dispone de una parte de la información de manera que deben combinarse los recursos de todos para que el grupo pueda alcanzar sus objetivos. En la interdependencia de roles los miembros tienen roles interconectados y complementarios. Por último, la interdependencia de la tarea se refiere a la distribución del trabajo que obliga a completar a cada miembro su parte para que otro miembro del grupo pueda realizar su parte.

Tabla 1.5. Tipos de interdependencia

\begin{tabular}{|l|l|}
\hline Resultados de interdependencia & Medios de interdependencia \\
\hline Interdependencia de objetivos & Interdependencia de recursos \\
Interdependencia de recompensas & $\begin{array}{l}\text { Interdependencia de roles } \\
\text { Interdependencia de la tarea }\end{array}$ \\
\hline
\end{tabular}


En definitiva, y de acuerdo con Johnson y Johnson, es el docente el responsable de estructurar y favorecer la interdependencia positiva en primer lugar, mediante el establecimiento de objetivos grupales, y en segundo lugar y a fin de poder completarla, con la implantación del reconocimiento grupal, la división de recursos y la distribución de roles complementarios.

\subsection{Estructura de participación grupal}

Durante el proceso de interacción se aprecian cambios en cuanto a los tipos de relación que se establecen entre los miembros de un grupo, puesto que la interacción social se regula en cada momento de acuerdo a unas normas que determinan lo que Erickson (1982) denomina estructura de participación. Dicha estructura está vinculada a los derechos y obligaciones de los participantes de manera que, según Erickson, es dinámica y cambiante. Además este autor distingue otro tipo de organización relacionada con la estructura académica de la tarea que depende de las características y la secuenciación de la tarea.

Sin embargo, otros autores (Damon y Phelps,1989) diferencian tres dimensiones estructurales de tipos de interacción grupal, en base a la igualdad (equality) de roles y a la mutualidad en la interacción (interactive structure), la tutoría, la colaboración y la cooperación. La tutoría se caracteriza por la relación entre dos alumnos que abordan la tarea con un nivel diferente de habilidad y con unos roles de tutor y tutorado claramente asimétricos y por tanto con una baja igualdad y una baja mutualidad dado que se trata de una interacción dominada por el tutor. En la cooperación, las habilidades de los alumnos son heterogéneas pero con márgenes de proximidad y una igualdad elevada en tanto que la relación general es de simetría. La mutualidad es mediana y depende de la competencia entre equipos, la distribución de responsabilidades y la recompensa extrínseca o intrínseca. Por último, en la colaboración los alumnos tienen habilidades similares y los roles, pese a que diferentes, son simétricos de manera que presentan una elevada igualdad y una también una alta mutualidad puesto que los sujetos contribuyen a la interacción en un plano de igualdad. La tabla 1.6. resume esta clasificación de interacciones en base a su calidad.

Tabla 1.6. Calidad de la interacción según su dimensión (Duran, 2002)

\begin{tabular}{|l|l|l|l|}
\hline & Tutoría & Cooperación & Colaboración \\
\hline $\begin{array}{l}\text { Igualdad } \\
\text { (simetría) }\end{array}$ & Baja & Elevada & Elevada \\
(asimétrica) & (simétrica) & Elevétrica) \\
\hline Mutualidad & Baja & Mediana & Elada \\
\hline
\end{tabular}


No obstante, tal como puntualiza Duran (2002), en la práctica esta distinción no es tan nítida porque, en primer lugar, en las situaciones de cooperación se dan elementos característicos de otras situaciones y, en segundo lugar, porque algunos de los rasgos esenciales de cada una de ellas pueden verse afectados por la falta de estructuración de las interacciones sociales. Por otra parte, Dillenbourg (1999) distingue entre simetría de acción, simetría de conocimiento y simetría de estatus. La simetría de acción hace referencia al grado en que los participantes comparten las acciones que llevan a cabo. La simetría de conocimiento se refiere al grado en que los participantes comparten el mismo nivel de conocimiento, a menudo este tipo de simetría se confunde con la heterogeneidad puesto que es posible que dos alumnos tengan el mismo grado de expertitud pero diferentes puntos de vista sobre la tarea. Por último, la simetría de estatus se relaciona con la posición del alumno respecto a la comunidad a la que pertenece.

Otra propuesta para caracterizar la naturaleza de la colaboración en la interacción entre iguales es el modelo de análisis de Kumpulainen y Kaartinen (2003) para describir a nivel de episodios el proceso social entre los estudiantes. Estos autores distinguen entre seis modos de actividad social: colaborativo, tutorial, argumentativo, conflictivo, dominante y confuso. El modo colaborativo se caracteriza por una participación igualitaria de los miembros del grupo. En el modo tutorial un alumno ayuda y asiste a otro. El modo argumentativo se relaciona con la presencia de conflictos sociales o cognitivos que deben resolverse con la argumentación, mientras que en el modo conflictivo los conflictos quedan sin resolver. En el modo dominante un estudiante controla el trabajo produciéndose una participación desigual. Finalmente, el modo confuso se caracteriza por la ausencia de comprensión compartida.

\section{TIC y aprendizaje cooperativo}

Desde los inicios de la incorporación de las TIC en las prácticas educativas escolares hasta la actualidad son diversas las propuestas de clasificación de usos educativos que se han llevado a cabo dependiendo, tal como señala Twining (2002), del marco conceptual sobre los procesos de enseñanza y aprendizaje y del uso de las TIC que se adopta como marco de referencia.

Entre dichas propuestas de clasificación cabe señalar la clásica diferenciación entre el ordenador como tutor, como herramienta y como alumno de Crook (1994) y la introducción del concepto mind tool desde una perspectiva cognitiva (Jonassen, 1995) que define las TIC como instrumentos que permiten que los alumnos re-presenten su conocimiento de diversas maneras y puedan reflexionar sobre él.

A menudo, algunas de las propuestas, alejadas de la observación de la práctica educativa en el aula, se han centrado en el tipo de software y en su diseño, ignorando las maneras en que éste se utiliza. Por contra, desde una perspectiva sociocognitiva, Barab et al. (2000) proponen un modelo centrado en la tecnología para facilitar la investigación del alumno y dar apoyo a los estudiantes para desarrollar "grounded 
constructions". Por otro lado, la propuesta de Twining (2002), calificada por Coll (2004) de neutral, diferencia entre el uso de apoyo, de extensión y de transformación en función de tres criterios relacionados con los contenidos, con los procesos y con la propia práctica desarrollada.

En nuestro país Coll et al. (2004), desde una perspectiva socioconstructivista y con el objetivo de superar diversas limitaciones de las clasificaciones mencionadas, elaboran un sistema de clasificación de usos de las TIC a partir de la función mediadora de las TIC de las relaciones entre los tres elementos que conforman el espacio conceptual del triángulo interactivo e identifican cinco categorías de usos. El primer tipo está relacionado con las TIC como instrumento de mediación de las relaciones entre los alumnos y el contenido o la tarea de aprendizaje. En el segundo, las TIC se consideran un instrumentos mediadores de las relaciones entre los profesores y contenidos (y tareas) de enseñanza y aprendizaje. En el tercer tipo, las TIC aparecen como instrumentos mediadores de las relaciones entre los profesores y alumnos o entre los alumnos. En cuarto lugar las TIC se consideran instrumentos mediadores de la actividad conjunta desplegada por profesores y alumnos durante la realización de las tareas o actividades de enseñanza y aprendizaje. Finalmente, en el quinto tipo de uso establecido, las TIC se consideran instrumentos configuradores de entornos o espacios de trabajo y de aprendizaje.

Tal como se aprecia en algunas de las propuestas (véase Jonassen, 1995 y Coll, 2004), las TIC pueden utilizarse para llevar a cabo actividades que específicamente requieran la cooperación. De ahí que su incorporación en situaciones de aprendizaje cooperativo haya implicado la aparición de nuevos contextos de interacción educativa caracterizados por la presencialidad o la virtualidad, por la sincronía o la asincronía. Conforme a la aparición de dichos escenarios, Crook (1996) establece cuatro tipos según el lugar que se otorga a las interacciones respecto a los ordenadores (interacciones en relación a los ordenadores, interacciones delante de ellos, interacciones en torno a ellos e interacciones mediante ellos).

El uso del ordenador como apoyo del aprendizaje cooperativo nos remite de manera indiscutible a la propuesta de Koschmann (1996) referida al CSCL-ComputerSupported Collaborative Learning- como paradigma que combina las nuevas tecnologías con el aprendizaje cooperativo. Desde este paradigma se considera que las TIC pueden ser utilizadas para facilitar, aumentar e incluso modificar las interacciones que tienen lugar entre los miembros de un grupo cooperativo. Además las aplicaciones diseñadas con dichos fines responden a diferentes usos en la propia aula o entre aulas conectando a usuarios, para la creación de aulas virtuales o bien para coordinar interacciones sincrónicas como chats o asincrónicas como el correo electrónico. En definitiva, se trata de utilizar la tecnología, teniendo en cuenta las diversas posibilidades del diseño instruccional y los aspectos psicoeducativos propios del aprendizaje cooperativo, para apoyar dicho aprendizaje.

En lo que concierne a la investigación, desde el paradigma CSCL el foco se centra en la calidad del proceso colaborativo, a saber, en la manera en que los alumnos intercambian la información, cómo discuten diferentes perspectivas y coordinan sus esfuerzos para resolver un problema juntos o el modo en que hacen uso de las herramientas tecnológicas de las que disponen. 
Además del CSCL, otros autores han centrado la investigación en el ámbito de las TIC y el aprendizaje cooperativo presencial. En este sentido, cabe mencionar a Wegerif (1996), miembro del equipo que desarrolla el proyecto "Thinking Together" dirigido a promover la mejora de las habilidades de pensamiento de los alumnos a través de lecciones de habla, que focaliza la atención en cómo el ordenador puede estimular y mejorar el habla de los alumnos y como consecuencia su razonamiento. En la misma línea de investigación Thompson (2005) se centra en el habla de los alumnos ante diferentes tareas con el ordenador (creando un Power Point, buscando información en Internet o creando imágenes).

En relación al ordenador como herramienta para la búsqueda colaborativa de información en Internet, Lazonder (2005) diferencia dos líneas de investigación. Una primera línea centrada en temas periféricos como por ejemplo el rol que adoptan los individuos en la búsqueda colaborativa de información (Prekop, 2002), y una segunda línea dirigida a diseñar herramientas de apoyo para fomentar actividades colaborativas de búsqueda (Chau, Zeng, Chen, Huang y Hendriawan, 2003).

De acuerdo con Lazonder (2005) existe un vacío de estudios que expliquen cómo los grupos se organizan y llevan a cabo el proceso de búsqueda colaborativa y proporcionen evidencias empíricas de las presuntas ventajas de la búsqueda colaborativa en Internet. A fin de contribuir a dicha necesidad Lazonder realiza un estudio comparando estudiantes individuales con parejas haciendo búsquedas en Internet y concluye que la regulación y la colaboración presentes en las parejas, aparecen como procesos que mejoran los resultados de búsqueda.

A fin de superar algunas de las limitaciones que, a nuestro modo de ver, tienen las líneas de investigación mencionadas, centradas únicamente en cómo el ordenador apoya la discusión o influye sobre el habla en la línea del grupo Thinking Together, situamos nuestra propuesta en otra línea de investigación dirigida a describir la naturaleza de la actividad social y cognitiva. Dicha línea de investigación, se centra tanto en la actividad discursiva, como en la cognitiva y colaborativa de los alumnos cuando trabajan en grupo con apoyo del ordenador (Kumpulainen, Salovaara y Mutanen, 2001). En otros términos y de acuerdo con nuestro punto de vista, se contempla de manera integrada la actividad mental constructiva del alumno y la interacción social con los compañeros.

\section{Situaciones educativas de aprendizaje cooperativo presencial con TIC}

En este apartado se presenta un posible escenario de aprendizaje cooperativo con TIC que a nuestro modo de ver resultaría adecuado para poder observar y estudiar los procesos psicoeducativos descritos en el primer apartado. Ante todo, cabe decir que se trata de un escenario educativo presencial perteneciente a la educación secundaria obligatoria en Cataluña desarrollado en su contexto natural sin intervención de los investigadores, ni en su diseño ni en su desarrollo. Con este objetivo se definen una 
serie de condiciones que el escenario educativo en cuestión debería reunir en relación a los alumnos, al profesor y al diseño de la actividad.

En relación a los alumnos, se considera oportuno que tengan experiencia en el trabajo cooperativo, esto es, que estén habituados a trabajar en grupos y a poner en funcionamiento las habilidades sociocognitivas propias de las interacciones en el sí de los grupos cooperativos. Además se espera que sean usuarios expertos en el uso educativo de las TIC.

En referencia al profesor, se espera que tenga formación en aprendizaje cooperativo así como en el uso educativo de las TIC, además sería conveniente que fuera un profesor con una experiencia media o elevada en la docencia del área curricular que imparte. En cuanto al papel del profesor durante el proceso de enseñanza y aprendizaje se espera que ofrezca ayudas educativas a los alumnos, ya sean verbales o en forma de pautas, en cada una de las fases que integran el proceso.

En cuanto al diseño de la actividad, sería deseable que facilite las condiciones necesarias para favorecer un alto nivel de interacción entre los miembros de los grupos y que la cooperación sea indispensable para lograr los objetivos de aprendizaje. Además, la demanda cognitiva debería incluir competencias relacionadas con el uso de la información y las TIC deberían tener un papel fundamental en este proceso, incluyendo la mayor diversidad de usos educativos posibles así como su presencia en la mayor parte del proceso de enseñanza y aprendizaje. En cuanto al número de ordenadores por grupo, teniendo en cuenta que los grupos están formados por tres o cuatro miembros sería oportuno que fuera menor que el número de alumnos, en este sentido hay que tener en cuenta que los ordenadores determinaran en gran medida la interacción que se establezca entre los alumnos de un mismo grupo.

Por otro lado, en lo que se refiere a los grupos cooperativos, serán heterogéneos formados por el profesor siguiendo los criterios que considere adecuados como el rendimiento académico o las habilidades sociales. Asimismo, se espera que sean los alumnos quienes determinen sus roles en el seno de cada grupo.

En cuanto a la evaluación, debería contemplarse la evaluación individual y grupal de la actividad, teniendo en cuenta los resultados reflejados en las producciones grupales y el proceso de trabajo grupal. En relación a éste último, se tendrán en cuenta las competencias comunicativas relacionadas con la planificación y el desarrollo de la tarea, por ejemplo si han compartido los objetivos de la tarea, si han establecido algunas normas de participación o cómo han solucionado los conflictos y superado los desacuerdos.

Finalmente, estimamos oportuno señalar que sería muy conveniente que durante el desarrollo de la secuencia didáctica se hiciera uso de una plataforma educativa que permitiera generar un entorno de aprendizaje de carácter público susceptible de ser utilizado por el profesor y los alumnos. El profesor podría utilizar la plataforma para presentar los contenidos preseleccionados (páginas web educativas), la planificación de la actividad y/o comunicarse con los alumnos más allá de las barreras físicas del aula ofreciéndoles feedback. Los alumnos, por su parte, podrían a su vez comunicarse 
con el profesor, seguir su proceso de evaluación y acceder a los espacios donde colgar las producciones grupales e individuales.

\section{Una propuesta de dimensiones de análisis}

En virtud de los procesos psicoeducativos descritos en el primer apartado y de las características de la situación educativa objeto de análisis se ha optado por incluir cinco dimensiones en nuestra propuesta de análisis. La primera de ellas, la gestión de la tarea, hace referencia a los procesos de regulación y a la interdependencia positiva, la segunda, construcción compartida de conocimiento, a los procesos de construcción conjunta de conocimiento, a los conflictos entre puntos de vista moderadamente divergentes y a la construcción de representaciones compartidas y la tercera, estructura de interacción grupal, a la estructura de participación grupal. Además, y directamente relacionadas con la presencia de las TIC en el proceso de enseñanza y aprendizaje, hemos añadido dos dimensiones, una en relación a los usos educativos de las TIC y una referente al nivel cognitivo en el uso de la información (véase la Figura 4.1.).

A continuación se expone en relación a cada una de las dimensiones propuestas, la redefinición que se ha llevado a cabo teniendo en cuenta la situación de análisis. Asimismo, al final del apartado se recoge en la Tabla 4.2. una propuesta de dimensiones de análisis con sus categorías correspondientes.

Tabla 4.1. Correspondencia entre procesos psicoeducativos descritos y propuesta de dimensiones de análisis.

\begin{tabular}{|c|c|}
\hline Procesos psicoeducativos descritos & Dimensiones de análisis propuestas \\
\hline Regulación a través del lenguaje & Gestión de la tarea \\
\hline Interdependencia positiva & $\begin{array}{r}\text { Construcción compartida de conocimiento } \\
\text { (proceso y resultados) } \\
\text { vista moderadamente divergentes }\end{array}$ \\
\cline { 1 - 1 } $\begin{array}{c}\text { Contrucción de representaciones } \\
\text { compartidas }\end{array}$ & \\
\hline Construcción compartida de conocimiento & \\
\hline Estructura de participación grupal & Estructura de participación grupal \\
\hline TIC & Usos educativos de las TIC \\
\cline { 2 - 2 } & Nivel cognitivo en el uso de la información \\
\hline
\end{tabular}




\subsection{Gestión de la tarea}

A pesar de que desde nuestro punto de vista los procesos de regulación del aprendizaje pertenecen tanto al plano interpsicológico como intrapsicológico, en la presente propuesta situamos esta dimensión de acuerdo con la perspectiva más reciente según la cual la regulación se considera un proceso grupal situado en el plano social o interpsicológico (liskala, Vauras y Lehtinen, 2004; Hurme, Palonen y Järvela, 2006).

Asimismo y en cuanto al foco de investigación, nos centramos en las intervenciones de los miembros del grupo que hacen referencia a las funciones de planificación, control y valoración de la tarea. Además, se considera la interdependencia positiva incluida en esta dimensión en la medida que en las intervenciones se haga referencia a la disposición y a la necesidad de los estudiantes de supervisar las acciones que llevan a cabo mediante la división de recursos y el establecimiento de roles complementarios a fin de alcanzar los objetivos grupales.

\subsection{Construcción compartida de conocimiento}

Puesto que entendemos el conocimiento compartido como las coincidencias y similitudes en las representaciones mentales sobre el contenido y al conocimiento común sobre los mismos conceptos como resultado del proceso de colaboración (Roschelle, 1992; Cannon-Bowers, Salas y Converse, 1993; Jeong y Chi, 1999 y Weinberger et al., 2007), nuestro interés se centra por un lado en cómo se desarrolla dicho proceso, en concreto se trata de analizar las intervenciones de los estudiantes, tanto de acción como de discurso, que hacen referencia al contenido de enseñanza y aprendizaje. Por otro lado nos centramos en el resultado de dicho proceso, a saber, en las representaciones mentales sobre el conocimiento que tienen los alumnos al finalizar el proceso de colaboración.

Después de la extensa revisión teórica y empírica llevada a cabo sobre diversas propuestas de análisis del proceso de construcción compartida de conocimiento, véase los estudios sobre el habla (Fisher, 1993; Wegerif y Mercer, 1997), las propuestas que parten de aspectos de contenido y función comunicativa (Kumpulainen y Mutanen, 1999; Fischer et al., 2002) y las propuestas de análisis de la interacción en entornos virtuales asincrónicos (Henri, 1992; Gunawardena et al., 1997; Putambekar, 2004; Garrison y Anderson, 2005), hemos adoptado como punto de partida el modelo de Gunawardena, Lowe y Anderson (1997). Puesto que este modelo de análisis está elaborado para un debate virtual en un entorno universitario, hemos adaptado la propuesta al tipo de situación educativa caracterizada en el apartado anterior, a saber, a las características de la tarea que nos ocupa y a la edad de los alumnos.

En relación a los resultados de dicho proceso, se propone analizar los productos grupales y las representaciones mentales individuales sobre el contenido obtenidas a través de un postest con el fin de poderlas comparar intragrupalmente y de esta manera identificar hasta qué punto o en qué grado los miembros de un mismo grupo 
comparten la representación mental sobre el contenido después del proceso de colaboración (Jeong y Chi, 1999; Fischer y Mandl, 2005).

\subsection{Estructura de interacción grupal}

Desde nuestro punto de vista estudiar la naturaleza social de la colaboración requiere estudiar la relación que se establece entre los participantes de acuerdo con el grado de simetría de acción que desarrollan (Dillenbourg, 1999). Como punto de partida y referencia teórica proponemos optar por los modelos propuestos por Damon y Phelps (1989) y Kumpulainen y Kaartinen (2003) que distinguen cuatro tipos de estructuras de interacción social caracterizadas por el grado ascendente de simetría de acción: individualista, dominante, tutorial y colaborativa (definidas en el apartado 1.6.). Ahora bien, se prevé la posibilidad de añadir nuevas categorías en función de la actividad que lleven a cabo los alumnos y los roles que adopten.

\subsection{Usos educativos de las TIC}

La inclusión de esta dimensión de análisis viene dada por las características descritas en el tercer apartado de la situación de análisis dónde el uso de las TIC es una constante en todo el proceso de enseñanza y aprendizaje. Nuestro planteamiento de usos educativos de las TIC parte de la propuesta establecida por Chen, Hsu y Hung (2000) que clasifican los usos educativos en cuatro categorías según sea su papel mediador en el proceso de enseñanza y aprendizaje. Estos autores distinguen entre las TIC como herramientas informativas, de situación, constructivas y comunicativas. Las herramientas informativas son aplicaciones que proporcionan a los alumnos información en diferentes formatos (texto, sonido, gráficos, video). Las herramientas de situación por su parte sitúan al alumno en entornos dónde pueden experimentar contextos concretos. Las constructivas permiten al alumno manipular la información y crear un producto y, por último, las herramientas comunicativas permiten la comunicación entre alumnos o entre alumnos y docentes más allá de las barreras físicas del aula.

\subsection{Nivel cognitivo en el uso de la información}

Dado que desde nuestro punto de vista el aprendizaje no sólo va a depender del proceso de construcción compartida de conocimiento en los grupos cooperativos sino que también va a influir el nivel cognitivo que se pone en juego cuando los alumnos manipulan la información, a saber, los contenidos de aprendizaje, consideramos fundamental estudiar cómo los alumnos manipulan la información y el nivel de procesamiento que alcanzan. 
La revisión empírica y teórica llevada a cabo sobre la actividad cognitiva nos ha llevado a diferenciar entre las propuestas propias de contextos sin tecnología que vinculan dicha actividad cognitiva con los procedimientos y las estrategias de aprendizaje, véase (Weinstein y Mayer, 1986; Pozo y Postigo, 1993; Monereo, 1994) y las pertenecientes a contextos de aprendizaje con tecnología que se concentran sobretodo en la descripción de las estrategias de navegación de los estudiantes (Barab et al., 1997; Wrigth, 1993).

Aunque las categorías que se han establecido parten de las propuestas elaboradas para contextos educativos sin tecnología, ha sido necesario tomar en consideración el papel de las TIC en la manipulación de los contenidos de aprendizaje y su relación directa con la demanda de la tarea, a fin de establecer categorías que correspondan a niveles sucesivos de complejidad cognitiva, del más bajo (copia literal) al más alto (establecer relaciones conceptuales).

TABLA 4.2. Propuesta de categorías para las dimensiones de análisis.

\begin{tabular}{|c|c|}
\hline & CATEGORÍAS \\
\hline \multirow{3}{*}{$\begin{array}{l}\text { GESTIÓN DE LA } \\
\text { TAREA }\end{array}$} & Planificación (PA) Planificación de la actividad \\
\hline & $\begin{array}{ll}\text { Control } & \text { (CA) Control de la actividad de los otros componentes } \\
& \text { (CP) Control de la propia actividad y conjunta }\end{array}$ \\
\hline & Valoración (VA) Valoración de la actividad \\
\hline \multirow{6}{*}{$\begin{array}{l}\text { ESTRUCTURA DE } \\
\text { INTERACCIÓN GRUPAL }\end{array}$} & $\begin{array}{ll}\text { Individualista } & \text { (I) Trabajo individual sin compartir ningún aspecto de la tarea ni del contenido. }\end{array}$ \\
\hline & (D) Un estudiante domina el trabajo \\
\hline & (T) Un estudiante ayuda y asiste al otro. \\
\hline & $\begin{array}{ll}\text { Colaborativa } & \text { (C) Los estudiantes participan de manera igualitaria en la actividad. }\end{array}$ \\
\hline & $\begin{array}{l}\begin{array}{c}\text { Complementaria } \\
\text { complementarios. }\end{array} \\
\text { (CM) }\end{array}$ \\
\hline & Trabajo en paralelo $(\mathbf{P})$ Los estudiantes realizan la misma tarea sin comunicarse \\
\hline \multirow[t]{4}{*}{ USOS TIC } & Herramienta informativa (HI) Las TIC proporcionan información en diferentes formatos. \\
\hline & Herramienta constructiva (HC) Las TIC se utilizan para manipular la información. Los alumnos producen un producto tangible. \\
\hline & Herramienta reproductiva (HR) Las TIC se utilizan para obtener una copia literal de otro formato diferente. \\
\hline & Herramienta comunicativa (HC) Las TIC permiten la comunicación entre profesor y alumnos más allá de las barreras físicas del aula. \\
\hline \multirow{4}{*}{$\begin{array}{l}\text { CONSTRUCCIÓN } \\
\text { COMPARTIDA DE } \\
\text { CONOCIMIENTO }\end{array}$} & 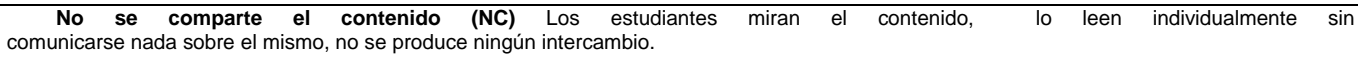 \\
\hline & $\begin{array}{l}\text { Comunicación del contenido (CO) Los estudiantes se limitan a decir, a informar sobre el contenido (leer en } \\
\text { en voz alta, dictar..) }\end{array}$ \\
\hline & $\begin{array}{l}\text { Discusión sobre el contenido (DC) Los estudiantes aportan información sobre el contenido acompañada de manifestaciones de acuerdo } \\
\text { o desacuerdo, dudas, opiniones, matices... }\end{array}$ \\
\hline & Compartir el conocimiento $\quad$ (CC) Los estudiantes establecen puntos de acuerdo sobre la definición del contenido. \\
\hline \multirow[t]{5}{*}{ NIVEL COGNITIVO } & $\begin{array}{l}\text { Copia literal/Reproducción (C-L) No hay selección de información, copian literalmente de los apuntes de los compañeros o de } \\
\text { documentos elaborados previamente en otros formatos. }\end{array}$ \\
\hline & Búsqueda (B) Los alumnos buscan información sobre el contenido. \\
\hline & $\begin{array}{l}\text { Selección- Copia literal (S-CL) Los alumnos distinguen y seleccionan los contenidos relevantes para } \\
\text { copiarlos literalmente. }\end{array}$ \\
\hline & Selección-parafraseo (S-P) Los alumnos seleccionan el contenido y lo modifican utilizando otras palabras. \\
\hline & Relaciones conceptuales (RC) Los alumnos jerarquizan, esquematizan o sintetizan los contenidos. \\
\hline
\end{tabular}




\section{Consideraciones finales}

De acuerdo con la revisión y posterior selección y adaptación de los procesos psicoeducativos implicados en el aprendizaje cooperativo a fin de establecer nuestra propuesta de dimensiones de análisis, a continuación se pone en evidencia dos aspectos a tener en cuenta en la investigación. El primero de ellos hace referencia a la metodología de análisis y el segundo al papel que se otorga a las TIC en prácticas educativas de educación formal.

En cuanto al primer aspecto, referido a aspectos metodológicos de análisis, el establecimiento de relaciones entre dimensiones nos permitirá caracterizar el funcionamiento de los diferentes grupos cooperativos con apoyo del ordenador teniendo en cuenta los procesos psicoeducativos implicados en el proceso de trabajo grupal, así como identificar las dificultades que obstaculizan el buen funcionamiento de un grupo cooperativo. Considerando este doble objetivo se prevé, por un lado, llevar a cabo un análisis secuencial de las categorías identificadas que nos permita encontrar posibles patrones de aparición en el aprendizaje cooperativo. Además, se identificarán diferentes segmentos de actividad, reconocidos por el observador por un cambio identificable y reconocible en la actividad, en los que se pondrán de manifiesto las diversas interrelaciones entre dimensiones. De ahí que podamos caracterizar cada uno de los segmentos identificados y obtener evidencias de si existe un orden o contingencia temporal significativa entre ellos.

Para ejemplificar algunas de las relaciones entre dimensiones a continuación exponemos algunas hipótesis al respecto. En principio esperamos que exista relación entre el uso educativo de las TIC y el nivel cognitivo en el uso de la información, así como entre la Gestión de la tarea y el uso educativo de las TIC. Sin embargo, no esperamos que exista una relación directa entre la Estructura de interacción grupal y el Uso educativo de las TIC. Asimismo, y en relación a los resultados, esperamos que los grupos con una presencia más elevada de categorías relacionadas con la Construcción compartida de conocimiento logren un nivel de aprendizaje de los contenidos superior reflejado en la calidad de los productos grupales.

El segundo aspecto a considerar, relacionado con el papel educativo de las TIC, requiere posicionarse en relación a la finalidad que se atribuye a las TIC en las prácticas educativas. Aunque las TIC disponen de unas características tecnológicas relativas a la manipulación y la comunicación información que las hacen potencialmente atractivas para el aprendizaje (véase Martí (1992), Coll y Martí (2001) y Coll (2003)) no son las TIC per se las que definen el aprendizaje sino las actividades que profesores y alumnos llevan a cabo con ellas. Tal como se desprende de algunas observaciones llevadas a cabo en las aulas, a menudo las TIC se utilizan en actividades que requieren habilidades cognitivas de bajo nivel como la reproducción, la copia o la recopilación de información. De acuerdo con Lim y Tay (2003), las TIC debieran servir para promover habilidades cognitivas de alto nivel en los alumnos como el diseño, la toma de decisiones y la solución de problemas. Habilidades que a su vez implican analizar, evaluar, conectar y elaborar. Obviamente, para que esto sea 
posible el profesor debe tener una formación adecuada que le permita tomar decisiones en el diseño de las actividades que estén orientadas a fomentar la autonomía del alumno y promueva dichas habilidades cognitivas complejas.

Todo ello nos remite de nuevo al currículum competencial definido por la LOE y en concreto a la competencia digital. Si el alumno, al finalizar la educación obligatoria debe ser competente en la utilización de las tecnologías de la información y la comunicación como instrumento de trabajo intelectual, el uso de las TIC en el aula debiera ser habitual y responder a la necesidad de formar al alumno, según la LOE, "en la capacidad de procesar y gestionar adecuadamente información abundante y compleja, resolver problemas reales, tomar decisiones, trabajar en entornos colaborativos ampliando los entornos de comunicación para participar en comunidades de aprendizaje formales e informales, y generar producciones responsables y creativas".

Dicho esto y siendo conscientes de la dificultad que supone en la práctica cumplir con estos objetivos, en nuestra opinión el estudio de las interrelaciones entre los procesos psicoeducativos y el uso de la TIC debiera llevarnos a entender mejor qué aspectos del uso de las TIC pueden producir cambios significativos tanto en el proceso de enseñanza y aprendizaje como en los resultados de dicho proceso. Además, y a fin de que dichos estudios sean útiles para la mejora de los procesos de enseñanza y aprendizaje, el conocimiento teórico que se derive de ellos debiera contribuir al diseño de los procesos de formación inicial y permanente del profesorado en el uso educativo de las TIC.

\section{Referencias bibliográficas}

AZMITIA, M. (1988). "Peer interaction and problem solving: When are two heads better than one?" Child Development. Núm. 59, pág. 87-96.

BARAB, S. A.; BOWDISH, B.E.; LAWLES, K.A. (1997). "Hypermedia navigation: profiles of hypermedia users". Educational Technology Research and Development. Núm. 45, pág. 23-21.

BARAB, S.A.; HAY, K. E.; DUFFY, T. (2000)."Grounded Constructions and How Technology Can Help". CRLT Technical Report. Pág. 12-00

BEARISON, D. J.; MAGZAMEN, S.; FILARDO, E. K. (1986). "Socio-conflict and cognitive growth in young children". Merrill-Palmer Quarterly. Núm. 32, pág. 51-72.

CANNON-BOWERS, J.A.; SALAS, E.; CONVERSE, S. (1993). "Shared mental models in expert team decision making". En: CASTELLAN, J.J. (ed.). Current Issues in Individual and Group Decision Making. Hillsdale, NJ: Erlbaum, pág. 221-246.

CLARK, H.H.; BRENNAN, S.E. (1991). "Grounding in communication". En: RESNICKL. B. y otros (eds.). Socially Shared Cognition, . Washington, DC: American Psychological Association, pág. 127-149 
COLL, C. (1984). "Estructura grupal, interacción entre alumnos y aprendizaje escolar". Infancia y Aprendizaje. Núm. 27-29, pág. 119-138.

COLL, C.; MIRAS, M. (1990). "La representación mútua profesor/alumno y sus repercusiones sobre la enseñanza y el aprendizaje". En COLL, C.; PALACIOS, J. y MARCHESI, A. (comps.). Desarrollo psicológico y educación, II. Psicología de la Educación. Madrid: Alianza.

COLL, C.; ONRUBIA, J. (1996). "La construcción de significados compartidos en el aula: actividad conjunta y dispositivos semióticos en el control y seguimiento mutuo entre profesor y alumnos". En

COLL, C.; MARTí, E. (2001). "La educación escolar ante las nuevas tecnologías de la información y la comunicación". En: C. COLL, J. PALACIOS y A. MARCHESI (Comps.), Desarrollo psicológico y educación 2. Psicología de la educación escolar. Madrid: Alianza, pág. 623-651).

COLL, C. (2003). "Tecnologies de la informació i la comunicació i pràctiques educatives". En: .COLL, C. (coord.). Psicologia de l'educació. Edición en formato web. Barcelona: Universitat Oberta de Catalunya.

COLL, C. (2004). "Psicología de la educación y prácticas educativas mediadas por las tecnologías de la información y la comunicación: una mirada constructivista". Sinéctica. Núm. 25, pág. 1-24

CROOK, Ch. (1998). Ordenadores y aprendizaje colaborativo. Madrid: Morata.

CHAU, M.; ZENG, D.; CHEN, H.; HUANG, M.; HENDRIAWAN, D. (2003). "Desing and development of a multi-agent collaborative web mining system". Decision Support Systems. Núm. 35, pág. 167-183

CHEN, D.T.; HSU, J.F.; HUNG, D. (2000). "Learning theories and IT: The computer as a tool". En: M.D. Williams (ed.), Integrating technology into teaching and learningconcept and applications Singapore: Prentice Hall. pág, 185-201.

DAMON, W.; PHELPS, E. (1989). "Critical distinctions among three approaches to peer education". International Journal of Educational Research. Núm. 58 (2), pág. 919.

DILLENBOURG, P.; BAKER, M.; BLAYE, A.; O'MALLEY, C. (1995). "The evolution of Research on Collaborative Learning". En: Learning in Humans and Machines. Oxford: Elsevier Science, pág. 189-211.

DILLENBOURG, P. (1999). Collaborative Learning. Cognitive and Computational Approaches. Oxford: Elsevier Science.

DOISE, W.; MUGNY, G.; PERRET-CLERMONT, A. (1975). "Social interaction and development of cognitive operations". European Journal of Social Psychology. Núm. 5 (3), pág. 367-383.

DURAN, D. (2002). "Tutoria entre iguals: processos cognitivorelacionals i anàlisi de la interactivitat en tutories fixes i recíproques". Tesis doctoral. Universitat Autònoma de Barcelona. [En línea] <http://www.tesisenred.net/TDX-1021103-181153/index_cs.html> [Fecha de consulta: 8 de noviembre de 2006] 
ELLIS, S.; KLAHR, D.; SIEGLER, R.S. (1993). "Effects of feedback and collaboration on changes in children's use of mathematical rules". Paper presentado en Society for Research in Child Development. New Orleans.

ERICKSON, F. (1982). "Classroom discourse as improvisation: Relationship between academic task structure and social participation structure in lessons". En: WILKINSON, L.C. (coord.), Communicating in the classroom. Nueva York: Academic Press, pág. 153-181.

FISHER, R. J. (1993). "Social Desirability Bias and the Validity of Indirect Questioning". Journal of Consumer Research. Núm. 20

FISCHER, F.; BRUHN, J.; GRÄSEL, C.; MANDL, H. (2002). "Fostering collaborative knowledge construction with visualization tools". Learning and Instruction. Núm. 12, pág. 213-232.

FISCHER F.; MANDL, H. (2005). "Knowledge convergence in computer-supported collaborative learning: the role of external representation tools". The Journal of the Learning Sciences. Núm. 14 pág. 405-441.

GABBERT, B.; JOHNSON, D.W.; JOHNSON, R.T. (1986). "Cooperative learning, group-to individual transfer, process gain, and the acquisition of cognitive reasoning strategies". The Journal of Psychology. Núm. 120 (3), pág. 265-278.

GARRISON, D.R.; ANDERSON, T. (2005). El e-learning en el siglo XXI: Investigación y práctica. Barcelona: Octaedro.

GROS, B. (2001). "Instructional design for computer supported collaborative learning in primary and secondary school". Computers and Human Behavior. Núm. 17 (5-6), pág. 439-451.

GUNAWARDENA, L.; LOWE, C.; ANDERSON, T. (1997). "Interaction analysis of a global on-line debate and the development of a contructivist interaction analysis model for computer conferencing". Journal of Education Computing Research. Núm.17 (4), pág. 395-429

HENRI, F. (1992). "Computer conferencing and content analysis". En: KAYE, I.R.(ed.), Collaborative learning through computer conferencing: The Najaden papers New York: Springer, pág. 115-136.

HOGAN, K.; NASTASI, B., PRESSLEY, M. (2000). "Discourse patterns and Collaborative Scientific Reasoning in Peer and Teacher-Guided discussions". Cognition and Instruction,. Núm. 17 (4), pág. 379-432.

HUME, T.; PALONEN, T.; JÄRVELA, S. (2006). "Metacognition in joint discussions: an analysis of the patterns of interaction and the metacognitive content of the networked discussions in Mathematics". Metacognition Learning. Núm. 1, pág. 181-200.

IISKALA, T.; VAURAS, M.; LETHINEN, E. (2004). "Socially-shared metacognition?". Hellenic Journal of Psychology. Núm. 1, pág. 147-178.

JEONG, H.; CHI, M.T.H. (1999). "Constructing Shared Knowledge During Collaboration and Learning". Poster presented at the AERA Annual Meeting, Montreal, Canada.

JEONG, H.; CHI, M.T.H. (2007). "Knowledge convergence and collaborative learning". Instructional Science. Núm. 35, pág. 287-315. 
JOHNSON, D.W.; JOHNSON, R.T(1990). Cooperation and competition: Theory and research. Lillsdale, NJ: Lawrance Erlbaum.

JOHNSON, D.W.; JOHNSON, R.T. (1992). "Positive interdependence: Key to effective cooperation". En: R. HERTZ-LAZAROWITZ y N. MILLER (Eds.), Interaction in cooperative groups: The theoretical anatomy of group learning New York: Cambridge University Press, pág. 174-199.

JOHNSON, D.W.; JOHNSON, R.T. (1999). Aprender juntos y solos. Aprendizaje cooperativo, competitivo e individualista. Buenos Aires: Aique.

JONASSEN, D.H. (1995). "Supporting Communities of Learners with Technology: A vision for Integrating Technology with Learning in Schools". Educational Technology, July-August, Pág. 60-63.

KOSCHMAN, T. (1996). CSCL: Theory and practice of an emerging paradigm. New Jersey: Lawrence Erlbaum Associates.

KUMPULAINEN, K.; MUTANEN, M. (1999). "The situated dynamics of peer group interaction: an introduction to an analytic framework". Learning and Instruction. Núm. 9 (5), pág. 449-473.

KUMPULAINEN, K.; KAARTINEN, S. (2003). "The Interpersonal Dynamics of Collaborative Reasoning in Peer Interactive Dyads". The Journal of Experimental Education. Núm. 71(4), pág. 333-370.

KUMPULAINEN, K.; SALOVAARA, H.; MUTANEN, M. (2001). "The nature of students' sociocognitive activity in handling and processing multimedia-based science material in a small group learning task". Instructional Science. Núm. 29, pág. 481-515.

LAZONDER, A. W. (2005). "Do two heads search better than one? Effects of student collaboration on web search behavior and search outcomes". British Journal of Educational Technology. Núm. 36 (3), pág. 465-475.

LIM, Ch. P.; TAY, L.Y. (2003). "Information and Communication Technologies (ICT) in an Elementary School: Students' Engagement in Higher Order Thinking". Journal of Educational Multimedia and Hypermedia, Núm. 12 (4), pág. 425-451.

MARTí, E. (1992). Aprender con ordenadores en la escuela. Barcelona: Horsori / ICE de la Universidad de Barcelona.

MELERO, M.; FERNÁNDEZ, P. (1995). "El aprendizaje entre iguales. El estado de la cuestión en Estados Unidos". En: La interacción social en contextos educativos. Madrid: Siglo XXI, pág. 35-56.

MERCER, N. (1997). La construcción guiada del conocimiento. El habla de profesores y alumnos. Barcelona: Paidós.

MONEREO, C. (Coord.); CASTELLÓ, M.; CLARIANA, M.; PALMA, M.; PÉREZ, M. L. (1994). Enseñanza y Aprendizaje. Barcelona: Graó.

POZO, J.I.; POSTIGO, Y. (1993). "Las estrategias de aprendizaje como un contenido del currículum". En: MONEREO, C. (comp.). Las estrategias de aprendizaje: procesos, contenidos e interacción. Barcelona: Edicions Doménech, pág. 105-112.

PREKOP, P. (2002). "A qualitative study of collaborative information seeking". Journal of Documentation. Núm. 58, pág. 533-547 
PUNTAMBEKAR, S. (2006). "Analysing collaborative interactions: divergence, shared understanding and construction of knowledge". Computers and Education. Núm. 47(3), pág. 332-351.

ROSCHELLE, J. (1992). "Learning by collaborating: Convergent conceptual change". The Journal of the Learning Sciences. Núm. 2 (3), pág. 235-276.

TEASLEY, S.D. (1992). "Communication and collaboration: The role of talk in children's peer collaboration". Tesis doctoral, University of Pittsburgh.

THOMPSON, J.C. (2005). "Cooperative Learning in Computer-Supported Classes". Tesis doctoral. The University of Melbourne. MAPS.

THOMPSON, L.; FINE, G.A. (1999). "Socially shared cognition, affect, and behavior: A review and integration". Personality \& Social Psychology Review. Núm. 3 (4), pág. 278-302.

TWINING, P. (2002). "Conceptualising Computer Use in Education. Introducing the Computer Practice Framework (CPF)". British Educational Research Journal. Núm. 28 (1), pág. 95-110.

SALOMON G.; PERKINS, D.N.; GLOBERSON, T. (1991). "Partners in Cognition: Extending Human Intelligence with Intelligent Technologies". Educational Researcher. Núm. 20(3), pág. 2-9.

SCHUNK, D. H.; ZIMMERMAN, J. (Eds.). (1994). Self-regulation of learning and performance. Issues and educational applications . Hillsdale, NJ: Erlbaum.

SLAVIN, R. (1980). "Cooperative learning". Review of Educational Research. Núm. 50 (2), pág. 315-342.

SLAVIN, R.E. (1990). Cooperative learning: Theory, research, and practice. New Jersey: Prentice Hall.

WEGERIF, R. (1996). "Using computers to help coach exploratory talk across the curriculum". Computers and Education. Núm. 26 (1-3), pág. 51-60.

WEGERIF, R.; MERCER, N. (1997). "Using Computer-based Text Analysis to Integrate Quantitative Methods in research on Collaborative Learning". Language and Education. Núm. 11 (4), pág. 271-286.

WEINSTEIN, C.E.; MAYER, R.F. (1986). "The teaching of learning strategies". En: WITTROCK, M.C. (ed.). Handbook of research on teaching. New York: McMillan, pág. 315-327.

WEINBERGER, A.; STEGMANN, K.; FISCHER, F. (2007). "Knowledge convergence in collaborative learning: Concepts and assessment". Leaning and Instruction. Núm. 17, pág. 416-426.

WERTSCH, J. (1988). Vygostky y la formación social de la mente (trad. De J. Zanón y M. Cortés). Barcelona: Paidós. [V.O.: Vygostky and the social formation of mind, Cambridge: Harvard University Press, 1985] 
WRIGHT, P. (1993). "To jump or not to jump: strategy selection while reading electronic texts". En: McKNIGHT, C.; DILLON A.; RICHARDSON, J. (eds.). Hipertext a Psychological Perspective. London: Ellis Horwood, pág. 137-152. 


\section{Resum}

La finalitat d'aquest article és presentar una proposta de dimensions d'anàlisi dels processos educatius implicats en l'aprenentatge cooperatiu, en un escenari educatiu amb un ús intensiu de les TIC. L'elaboració d'aquesta proposta implica, en primer lloc, la revisió teòrica prèvia dels processos psicoeducatius que expliquen la eficàcia de l'aprenentatge cooperatiu i, en segon Iloc, la definició d'un possible escenari on aplicar aquesta proposta d'anàlisi. Una vegada identificats aquests processos i descrit el mencionat escenari disposarem d'un marc teòric i pràctic del qual partir per prendre decisions relatives a les dimensions d'anàlisi que incloem en la nostra proposta.

\section{Paraules clau}

Aprenentatge cooperatiu, competències bàsiques, usos educatius de les TIC, processos psicoeducatius 


\section{Abstract}

The aim of this article is to propose dimensions for analysing psycho-educational processes involved in co-operative learning - in an educative setting where intensive use of ICT is practiced. This implies, in first place a theoretical revision of psychoeducational processes that explain the effectiveness of the cooperative learning process and in second place, the definition of a possible educational setting where this analysis can be applied. Once these processes and settings have been identified and defined we will be able to develop a conceptual and practical framework - which will aid us to make decisions in relation to the dimensions of analysis included in our proposal.

\section{Paraules clau}

Cooperative learning, basic competencies, ICT educational uses, psycho-educational processes 


\section{Lorena Becerril}

Ibecerril@uoc.edu

Doctorado en Sociedad de la Información y el Conocimiento

\section{Lorena Becerril}

Ibecerril@uoc.edu

Doctorat en Societat de la Informació i el Coneixement

\section{Lorena Becerril}

Ibecerril@uoc.edu

Information and knowledge Society Doctoral Programme

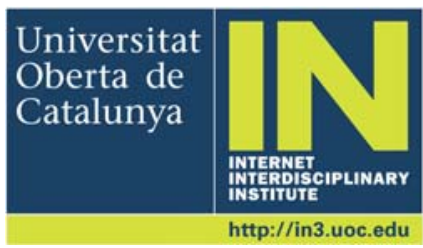

